\title{
Photocatalytic Alkylation of $\alpha$-(Trifluoromethyl)Styrenes with Potassium Xanthogenates
}

\author{
Vyacheslav I. Supranovich and Alexander D. Dilman *
}

check for

updates

Citation: Supranovich, V.I.; Dilman, A.D. Photocatalytic Alkylation of $\alpha$-(Trifluoromethyl)Styrenes with Potassium Xanthogenates. Catalysts 2021, 11, 1555. https://doi.org/ $10.3390 /$ catal11121555

Academic Editor: Ken-ichi Fujita

Received: 1 December 2021

Accepted: 15 December 2021

Published: 20 December 2021

Publisher's Note: MDPI stays neutral with regard to jurisdictional claims in published maps and institutional affiliations.

Copyright: (c) 2021 by the authors. Licensee MDPI, Basel, Switzerland. This article is an open access article distributed under the terms and conditions of the Creative Commons Attribution (CC BY) license (https:// creativecommons.org/licenses/by/ $4.0 /)$.
N. D. Zelinsky Institute of Organic Chemistry, Leninsky Prosp. 47, 119991 Moscow, Russia; supranv@ioc.ac.ru

* Correspondence: dilman@ioc.ac.ru

\begin{abstract}
A protocol for the coupling of potassium xanthogenates with $\alpha$-(trifluoromethyl)styrenes in the presence of triethyl phosphite is reported. The reaction is carried out under blue light irradiation in the presence of organic photocatalyst 3DPAFIPN. The reaction proceeds via formation of alkyl radicals from readily available xanthogenate salts via oxidative desulfurization and cleavage of the carbon-oxygen bond assisted by triethyl phosphite.
\end{abstract}

Keywords: radical reactions; xanthogenates; photocatalysis; triethyl phosphite

\section{Introduction}

Radical reactions constitute a rapidly developing branch of synthetic organic chemistry [1]. The advent of photocatalysis has offered novel opportunities in radical chemistry, primarily associated with methods for the generation of radical intermediates [2-4]. Recently, we described an approach for the direct generation of free radicals from aliphatic thiols via their in situ conversion into zinc thiolates with subsequent abstraction of the sulfur by triphenylphosphine [5] (Scheme 1) The key idea of this method is that a combination of a thiolate and a phosphorus (III) reagent upon photoredox oxidation leads to a free radical and the strong phosphorus-sulfur double bond. This reaction may proceed via initial oxidation of either the thiolate anion or the phosphorus atom. Herein, we report the extension of this principle to alcohols, which are far more abundant than thiols. We proposed that potassium xanthogenates, which can be easily obtained from alcohols, could be involved in reaction with phosphorus (III) reagents under photocatalytic conditions. Indeed, after abstraction of the sulfur by the phosphorus reagent, the thiocarbonyl radical is formed, which is prone to expel carbonyl sulfide (COS) with the generation of alkyl radical [6,7]. As coupling partners, we employed $\alpha$-(trifluoromethyl)styrenes, since they are known to be effective radical acceptors leading, after redox cycle, to gem-difluorinated styrenes [8,9]. During the preparation of this manuscript we became aware of a similar concept, with the alkyl radicals being trapped by acrylates [10].

Previous work:
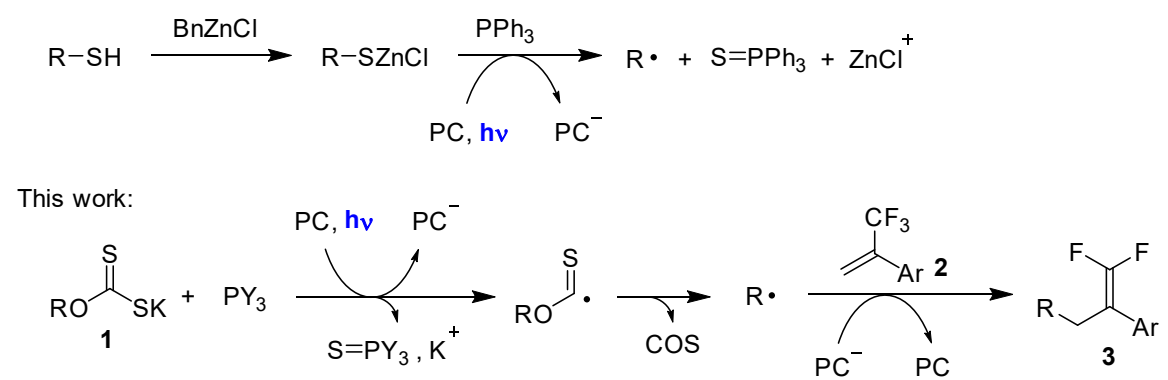

Scheme 1. Generation of radicals from sulfur derivatives. 


\section{Results}

Potassium xanthogenate 1a derived from phenethyl alcohol was used as a model reagent and its coupling with styrene $2 \mathbf{a}$ under blue light irradiation was carried out. We performed extensive variation of the phosphorus (III) reagent, photocatalyst, solvent, and the reaction time (Table 1). Using triphenylphosphine as a reagent and a typical iridium catalyst, the best result was achieved in dichloromethane as solvent within $1.5 \mathrm{~h}$ (entry 3). The switch to an organic photocatalyst 3DPAFIPN (0.6 mol\%) [11] led to further improvement. Rewardingly, triethyl phosphite also proved to be a suitable reagent. With this phosphite, the complete conversion of styrene $2 \mathrm{a}$ was achieved in $3 \mathrm{~h}$, and gave product $3 a$ in a $68 \%$ isolated yield (entry 16$)$.

Table 1. Optimization studies.

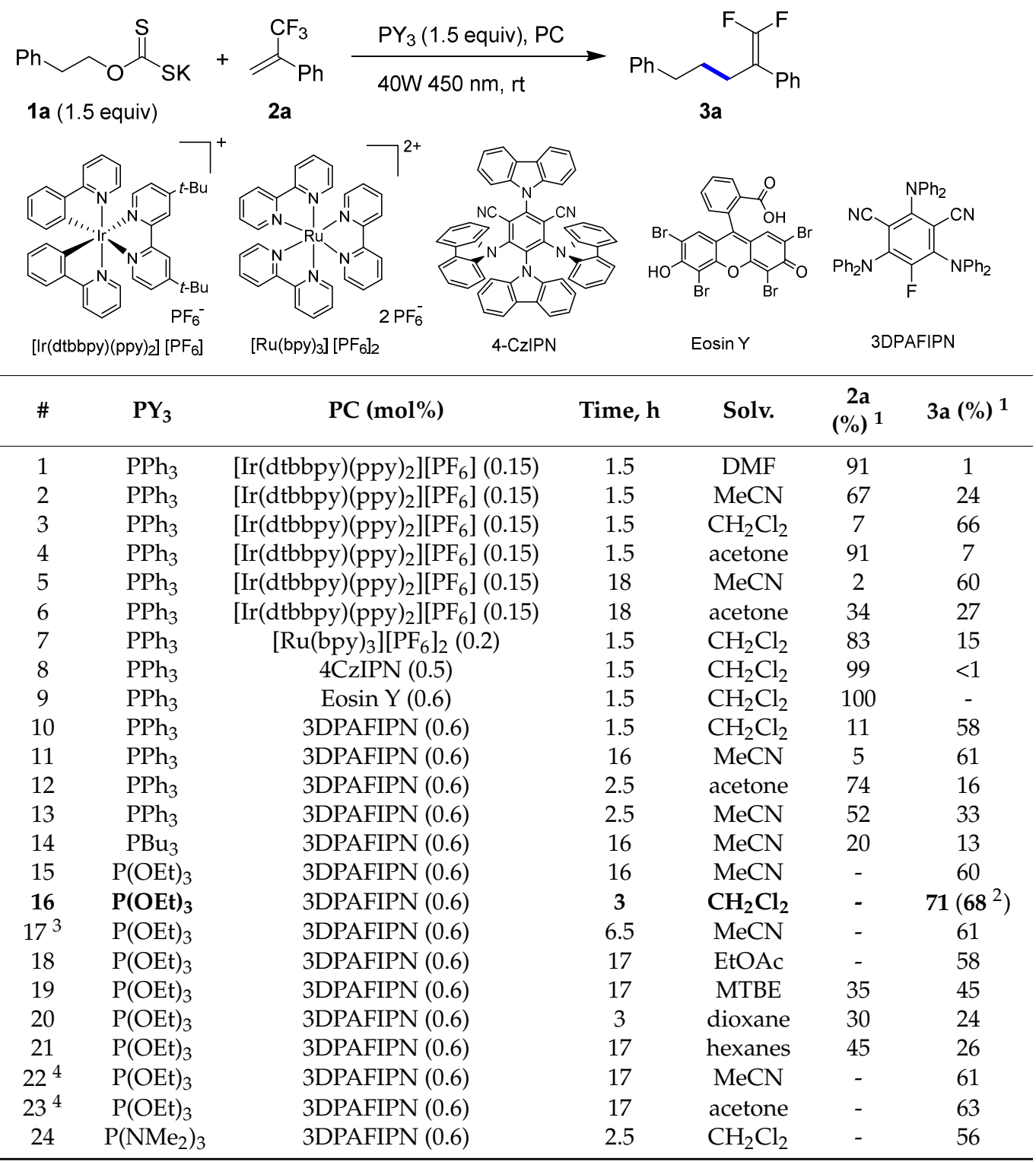

${ }^{1}$ Determined by GC analysis; ${ }^{2}$ isolated yield; ${ }^{3} 2.5$ equiv of $\mathrm{P}(\mathrm{OEt})_{3} ;{ }^{4} 6.0$ equiv of $\mathrm{P}(\mathrm{OEt})_{3}$.

Under the optimized conditions, potassium xanthogenates 1 were combined with $\alpha$-(trifluoromethyl)styrenes 2 (Scheme 2). The reaction involving generation of primary radicals worked well, while xanthogenates generating secondary radicals required five equivalents of triethyl phosphite. In this way, cyclopentyl, cyclohexyl, isopropyl, and tert-butyl radicals were successfully intercepted by styrene $\mathbf{1 a}$. 

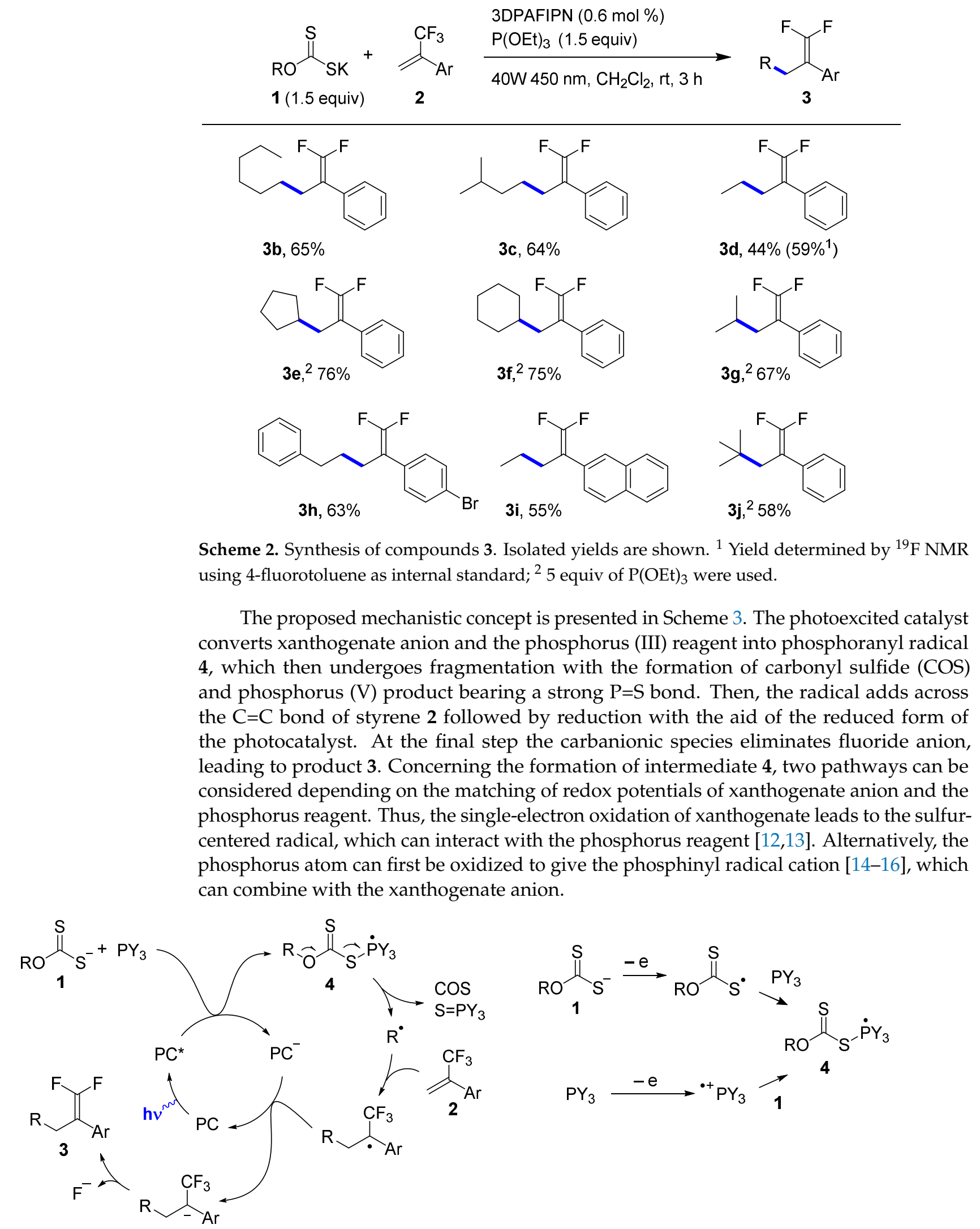

Scheme 3. Proposed mechanism. 


\section{Materials and Methods}

\subsection{General Information}

Dichloromethane was distilled from calcium hydride and stored over $3 \AA$ molecular sieves. Column chromatography was carried out using silica gel (230-400 mesh). For TLC, precoated plates F-254 were used. NMR spectra were recorded on a Bruker Avance II 300 instrument (see Supplementary Materials for copies of NMR spectra). HRMS spectra were measured using electrospray ionization and time-of-flight analyzer. The measurements were performed in positive $(4500 \mathrm{~V})$ or negative $(3200 \mathrm{~V})$ ion modes; $\mathrm{m} / \mathrm{z}$ mass range from 50 to 3000 .

Starting compounds were prepared according to literature procedures: $\alpha$-(trifluoromethyl)styrenes [17]; potassium phenethyl xanthogenate [18]; potassium $n$-hexyl xanthogenate [19]; potassium isoamyl xanthogenate [20]; cyclopentyl xanthogenate [20]; potassium cyclohexyl xanthogenate [20]; potassium isopropyl xanthogenate [21]; potassium tert-butyl xanthogenate [22]; 2,4,6-tris(diphenylamino)-5-fluoroisophthalonitrile (3DPAFIPN) [11].

\subsection{Reaction of Potassium Xanthagenates with $\alpha$-(Trifluoromethyl)styrenes (General Procedure)}

Potassium xanthagenate 1 ( $0.75 \mathrm{mmol}, 1.5$ equiv), and 3DPAFIPN $(2.0 \mathrm{mg}, 0.6 \mathrm{~mol} \%)$ were placed in a test tube (Duran \# 261351155, Roth \# K248.1, outside diameter $12 \mathrm{~mm}$ ). The tube was evacuated and filled with argon. Dichloromethane $(2.0 \mathrm{~mL})$, triethyl phosphite (for $\mathbf{3 a}-\mathbf{d}, \mathbf{h}, \mathbf{i}, 128 \mu \mathrm{L}, 0.75 \mathrm{mmol}, 1.5$ equiv; for $3 \mathbf{e}-\mathbf{g}, \mathbf{j} 431 \mu \mathrm{L}, 2.5 \mathrm{mmol}, 5.0$ equiv), and $\alpha$-(trifluoromethyl)styrene 2 ( $0.5 \mathrm{mmol}, 1.0$ equiv) were added. The tube was tightly closed with a screw cap and irradiated for $3 \mathrm{~h}$ by blue light. For the irradiation, a $450 \mathrm{~nm}$ LED chip (Hontiey royal blue $100 \mathrm{~W}$, operated at $40 \mathrm{~W}$ ) was employed. The distance between the LED chip and the test tube was $1 \mathrm{~cm}$. During irradiation, the tube was immersed into a water bath $\left(15-20^{\circ} \mathrm{C}\right)$. For the work-up, saturated $\mathrm{K}_{2} \mathrm{CO}_{3}(1 \mathrm{~mL})$ was added, the mixture was stirred for $15 \mathrm{~min}$, then water $(10 \mathrm{~mL})$ was added, and the mixture was extracted with hexanes $(3 \times 4 \mathrm{~mL})$. The combined organic layers were dried over sodium sulfate, filtered, concentrated. The crude material was purified by column chromatography on silica gel.

(5,5-Difluoropent-4-ene-1,4-diyl)dibenzene (3a) [5]. Yield $88 \mathrm{mg}(68 \%)$. Colorless oil. Chromatography: hexanes. $\mathrm{R}_{\mathrm{f}} 0.28$ (hexanes). ${ }^{1} \mathrm{H}$ NMR $\left(300 \mathrm{MHz}, \mathrm{CDCl}_{3}\right) \delta: 7.48-7.16$ $(\mathrm{m}, 10 \mathrm{H}), 2.70(\mathrm{t}, J=7.8,2 \mathrm{H}), 2.57-2.50(\mathrm{~m}, 2 \mathrm{H}), 1.85-1.74(\mathrm{~m}, 2 \mathrm{H}) .{ }^{13} \mathrm{C}\left\{{ }^{1} \mathrm{H}\right\} \mathrm{NMR}(75 \mathrm{MHz}$, $\left.\mathrm{CDCl}_{3}\right) \delta: 153.8(\mathrm{t}, J=288.6 \mathrm{~Hz}), 142.0,133.8,128.6,128.5,128.4,128.4(\mathrm{t}, J=3.3 \mathrm{~Hz}), 127.4$, 126.0, $92.4(\mathrm{t}, J=17.4 \mathrm{~Hz}), 35.4,29.5(\mathrm{t}, J=2.5 \mathrm{~Hz}), 27.5(\mathrm{t}, J=1.3 \mathrm{~Hz}) .{ }^{19} \mathrm{~F}$ NMR $(282 \mathrm{MHz}$, $\left.\mathrm{CDCl}_{3}\right) \delta:-92.1-92.5(\mathrm{~m}, 2 \mathrm{~F})$.

(1,1-Difluoronon-1-en-2-yl)benzene (3b). Yield $77 \mathrm{mg}(65 \%)$. Colorless oil. Chromatography: hexanes. $\mathrm{R}_{\mathrm{f}} 0.55$ (hexanes). ${ }^{1} \mathrm{H}$ NMR $\left(300 \mathrm{MHz} \mathrm{CDCl}_{3}\right) \delta: 7.46-7.28(\mathrm{~m}, 5 \mathrm{H}), 2.50-2.40$ $(\mathrm{m}, 2 \mathrm{H}), 1.51-1.21(\mathrm{~m}, 10 \mathrm{H}), 0.93(\mathrm{t}, J=7.0 \mathrm{~Hz}, 3 \mathrm{H}) .{ }^{13} \mathrm{C}\left\{{ }^{1} \mathrm{H}\right\} \mathrm{NMR}\left(75 \mathrm{MHz}, \mathrm{CDCl}_{3}\right) \delta: 153.7$ $(\mathrm{dd}, J=288.4,287.4 \mathrm{~Hz}), 134.1(\mathrm{~d}, J=1.7 \mathrm{~Hz}), 128.5,128.4(\mathrm{t}, J=3.3 \mathrm{~Hz}), 127.3,92.7(\mathrm{dd}$, $J=18.3,15.9 \mathrm{~Hz}), 32.0,29.16,29.12,27.9(\mathrm{t}, J=2.5 \mathrm{~Hz}), 27.8(\mathrm{t}, J=1.2 \mathrm{~Hz}), 22.8,14.2 .{ }^{19} \mathrm{~F}$ NMR $\left(282 \mathrm{MHz}, \mathrm{CDCl}_{3}\right) \delta:-92.9$ (s, 2F). HRMS (ESI): calcd for $\mathrm{C}_{15} \mathrm{H}_{20} \mathrm{~F}_{2} \mathrm{Ag}(\mathrm{M}+\mathrm{Ag}) 345.0579$, found 345.0566 .

(1,1-Difluoro-6-methylhept-1-en-2-yl)benzene (3c). Yield $72 \mathrm{mg}(64 \%)$. Colorless oil. Chromatography: hexanes. $\mathrm{R}_{\mathrm{f}} 0.55$ (hexanes). ${ }^{1} \mathrm{H}$ NMR $\left(300 \mathrm{MHz}, \mathrm{CDCl}_{3}\right) \delta: 7.46-7.28(\mathrm{~m}, 5 \mathrm{H})$, 2.48-2.39 (m, 2H), 1.64-1.48 (m, 1H), 1.48-1.36 (m, 2H), 1.30-1.20 (m, 2H), $0.89(\mathrm{~d}, J=6.6 \mathrm{~Hz}$, $\left.6 \mathrm{H}) .{ }^{13} \mathrm{C}_{\{}{ }^{1} \mathrm{H}\right\} \mathrm{NMR}\left(75 \mathrm{MHz}, \mathrm{CDCl}_{3}\right) \delta: 153.8(\mathrm{dd}, J=288.8,287.5 \mathrm{~Hz}), 134.1(\mathrm{~d}, J=1.9 \mathrm{~Hz})$, $128.5,128.4(\mathrm{t}, J=3.2 \mathrm{~Hz}), 127.3,92.7(\mathrm{dd}, J=18.6,15.9 \mathrm{~Hz}), 38.5,28.1(\mathrm{t}, J=1.2 \mathrm{~Hz}), 27.9$, $25.7(\mathrm{t}, J=2.5 \mathrm{~Hz}), 22.7 .{ }^{19} \mathrm{~F}$ NMR $\left(282 \mathrm{MHz}, \mathrm{CDCl}_{3}\right) \delta:-92.9(\mathrm{~s}, 2 \mathrm{~F})$. HRMS (ESI): calcd for $\mathrm{C}_{14} \mathrm{H}_{18} \mathrm{~F}_{2} \mathrm{Ag}(\mathrm{M}+\mathrm{Ag}) 331.0422$, found 331.0412 .

(1,1-Difluoropent-1-en-2-yl)benzene (3d) [23]. Yield $40 \mathrm{mg}$ (44\%). Colorless oil. Chromatography: hexanes. $\mathrm{R}_{\mathrm{f}} 0.50$ (hexanes). ${ }^{1} \mathrm{H}$ NMR $\left(300 \mathrm{MHz}, \mathrm{CDCl}_{3}\right) \delta: 7.44-7.28(\mathrm{~m}, 5 \mathrm{H})$, $2.43(\mathrm{tt}, J=7.5,2.4 \mathrm{~Hz}, 2 \mathrm{H}), 1.44(\mathrm{sept}, J=7.5 \mathrm{~Hz}, 1 \mathrm{H}), 0.95(\mathrm{t}, J=7.4 \mathrm{~Hz}, 3 \mathrm{H}) .{ }^{13} \mathrm{C}\left\{{ }^{1} \mathrm{H}\right\} \mathrm{NMR}$ $\left(75 \mathrm{MHz}, \mathrm{CDCl}_{3}\right) \delta: 153.9(\mathrm{dd}, J=288.9,286.6 \mathrm{~Hz}), 134.1(\mathrm{dd}, J=3.3,1.7 \mathrm{~Hz}), 128.5,128.5$ $(\mathrm{t}, J=3.3 \mathrm{~Hz}), 127.3,92.4(\mathrm{dd}, J=20.4,14.3 \mathrm{~Hz}), 29.8,21.1(\mathrm{t}, J=2.6 \mathrm{~Hz}), 13.5 .{ }^{19} \mathrm{~F}$ NMR $\left(282 \mathrm{MHz}, \mathrm{CDCl}_{3}\right) \delta:-92.8(\mathrm{~d}, J=45.1 \mathrm{~Hz}, 1 \mathrm{~F}),-92.9(\mathrm{~d}, J=45.1 \mathrm{~Hz}, 1 \mathrm{~F})$. 
(3-Cyclopentyl-1,1-difluoroprop-1-en-2-yl)benzene (3e) [5]. Yield $84 \mathrm{mg}$ (76\%). Colorless oil. Chromatography: hexanes. $\mathrm{R}_{\mathrm{f}} 0.55$ (hexanes). ${ }^{1} \mathrm{H}$ NMR $\left(300 \mathrm{MHz}, \mathrm{CDCl}_{3}\right)$

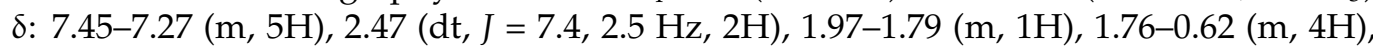
1.60-1.47 (m, 2H), 1.29-1.19 (m, 2H). ${ }^{13} \mathrm{C}\left\{{ }^{1} \mathrm{H}\right\}$ NMR $\left(75 \mathrm{MHz}, \mathrm{CDCl}_{3}\right) \delta: 154.1(\mathrm{dd}, J=289.5$, $286.3 \mathrm{~Hz}), 134.3(\mathrm{dd}, J=3.7,2.3 \mathrm{~Hz}), 128.6(\mathrm{t}, J=3.1 \mathrm{~Hz}), 128.5,127.3,92.5(\mathrm{dd}, J=21.2$, $13.5 \mathrm{~Hz}), 38.4(\mathrm{t}, J=2.4 \mathrm{~Hz}), 33.8(\mathrm{~d}, J=1.2 \mathrm{~Hz}), 32.3,25.2 .{ }^{19} \mathrm{~F} \mathrm{NMR}\left(282 \mathrm{MHz}, \mathrm{CDCl}_{3}\right) \delta$ : $-93.0(\mathrm{~d}, J=45.3 \mathrm{~Hz}, 1 \mathrm{~F}),-93.3(\mathrm{~d}, J=45.3 \mathrm{~Hz}, 1 \mathrm{~F})$.

(3-Cyclohexyl-1,1-difluoroprop-1-en-2-yl)benzene (3f) [5]. Yield $88 \mathrm{mg}$ (75\%). Colorless oil. Chromatography: hexanes. $\mathrm{R}_{\mathrm{f}} 0.55$ (hexanes). ${ }^{1} \mathrm{H}$ NMR $\left(300 \mathrm{MHz}, \mathrm{CDCl}_{3}\right) \delta: 7.45-7.28(\mathrm{~m}$, $5 \mathrm{H}), 2.35(\mathrm{dt}, J=7.2,2.4 \mathrm{~Hz}, 2 \mathrm{H}), 1.80-1.64(\mathrm{~m}, 5 \mathrm{H}), 1.41-1.26(\mathrm{~m}, 1 \mathrm{H}), 1.26-1.11(\mathrm{~m}, 3 \mathrm{H})$, 1.06-0.91 (m, 2H). ${ }^{13} \mathrm{C}\left\{{ }^{1} \mathrm{H}\right\}$ NMR $\left(75 \mathrm{MHz}, \mathrm{CDCl}_{3}\right) \delta: 154.2(\mathrm{dd}, J=290.3,285.9 \mathrm{~Hz}), 134.4$ $(\mathrm{dd}, J=4.5,2.8 \mathrm{~Hz}), 128.51,128.47(\mathrm{t}, J=3.2 \mathrm{~Hz}), 127.2,91.3(\mathrm{dd}, J=21.8,12.9 \mathrm{~Hz}), 35.9(\mathrm{t}$, $J=2.4 \mathrm{~Hz}), 35.4(\mathrm{~d}, J=1.3 \mathrm{~Hz}), 33.1,26.6,26.2 .{ }^{19} \mathrm{~F} \mathrm{NMR}\left(282 \mathrm{MHz}, \mathrm{CDCl}_{3}\right) \delta:-91.2(\mathrm{~d}$, $J=44.4 \mathrm{~Hz}, 1 \mathrm{~F}),-92.6(\mathrm{~d}, J=44.4 \mathrm{~Hz}, 1 \mathrm{~F})$.

(1,1-Difluoro-4-methylpent-1-en-2-yl)benzene (3g). [24] Yield $66 \mathrm{mg}(67 \%)$. Colorless oil. Chromatography: hexanes. $\mathrm{R}_{\mathrm{f}} 0.55$ (hexanes). ${ }^{1} \mathrm{H}$ NMR $\left(300 \mathrm{MHz}, \mathrm{CDCl}_{3}\right) \delta: 7.45-7.27(\mathrm{~m}$, $5 \mathrm{H}), 2.38-2.29(\mathrm{~m}, 2 \mathrm{H}), 1.73-1.56(\mathrm{~m}, 1 \mathrm{H}), 0.95(\mathrm{~d}, J=6.7 \mathrm{~Hz}, 6 \mathrm{H}) .{ }^{13} \mathrm{C}\left\{{ }^{1} \mathrm{H}\right\} \mathrm{NMR}(75 \mathrm{MHz}$, $\left.\mathrm{CDCl}_{3}\right) \delta: 154.3(\mathrm{dd}, J=289.8,286.2 \mathrm{~Hz}), 134.3(\mathrm{dd}, J=4.2,2.7 \mathrm{~Hz}), 128.5,128.5(\mathrm{t}, J=3.1 \mathrm{~Hz})$, 127.3, $91.9(\mathrm{dd}, J=21.6,13.2 \mathrm{~Hz}), 36.8(\mathrm{~d}, J=1.2 \mathrm{~Hz}), 26.6(\mathrm{t}, J=2.5 \mathrm{~Hz}), 22.2 .{ }^{19} \mathrm{~F}$ NMR $\left(282 \mathrm{MHz}, \mathrm{CDCl}_{3}\right) \delta:-92.5(\mathrm{~d}, J=44.2 \mathrm{~Hz}, 1 \mathrm{~F}),-92.9(\mathrm{~d}, J=44.2 \mathrm{~Hz}, 1 \mathrm{~F})$.

1-Bromo-4-(1,1-difluoro-5-phenylpent-1-en-2-yl)benzene (3h). Yield $106 \mathrm{mg}$ (63\%). Colorless oil. Chromatography: from hexanes to hexanes/EtOAc 50/1. $\mathrm{R}_{\mathrm{f}} 0.50$ (hexanes/EtOAc, 50/1). ${ }^{1} \mathrm{H}$ NMR $\left(300 \mathrm{MHz}, \mathrm{CDCl}_{3}\right) \delta: 7.53(\mathrm{~d}, J=8.5 \mathrm{~Hz}, 2 \mathrm{H}), 7.37-7.15(\mathrm{~m}, 7 \mathrm{H}), 2.67(\mathrm{t}$, $J=7.7,2 \mathrm{H}), 2.34(\mathrm{tt}, J=7.7,2.3,2 \mathrm{H}), 1.81-1.69(\mathrm{~m}, 2 \mathrm{H}) .{ }^{13} \mathrm{C}\left\{{ }^{1} \mathrm{H}\right\} \mathrm{NMR}\left(75 \mathrm{MHz}, \mathrm{CDCl}_{3}\right) \delta$ : $153.6(\mathrm{tt}, J=290.7,288.3 \mathrm{~Hz}), 141.7,132.7(\mathrm{dd}, J=3.4,1.9 \mathrm{~Hz}), 131.8,130.0(\mathrm{t}, J=3.3 \mathrm{~Hz})$, 128.48, 128.46, 126.0, 121.4, $91.7(\mathrm{dd}, J=21.0,14.1 \mathrm{~Hz}), 35.3,29.4(\mathrm{t}, J=2.4 \mathrm{~Hz}), 27.2(\mathrm{t}$, $J=1.1 \mathrm{~Hz}) .{ }^{19} \mathrm{~F} \mathrm{NMR}\left(282 \mathrm{MHz}, \mathrm{CDCl}_{3}\right) \delta:-91.2(\mathrm{~d}, J=42.1 \mathrm{~Hz}, 1 \mathrm{~F}),-91.4(\mathrm{~d}, J=42.1 \mathrm{~Hz}$, 1F). HRMS (ESI): calcd for $\mathrm{C}_{17} \mathrm{H}_{15} \mathrm{BrF}_{2} \mathrm{Ag}(\mathrm{M}+\mathrm{Ag}) 442.9371$, found 442.9363 .

2-(1,1-Difluoropent-1-en-2-yl)naphthalene (3i). Yield $64 \mathrm{mg}$ (55\%). Colorless oil. Chromatography: hexanes. $\mathrm{R}_{\mathrm{f}} 0.34$ (hexanes). ${ }^{1} \mathrm{H}$ NMR $\left(300 \mathrm{MHz}, \mathrm{CDCl}_{3}\right) \delta: 7.90-7.80(\mathrm{~m}, 4 \mathrm{H})$, $7.55-7.45(\mathrm{~m}, 3 \mathrm{H}), 2.53(\mathrm{tt}, J=7.4,2.3 \mathrm{~Hz}, 2 \mathrm{H}), 1.48(\mathrm{sept}, J=7.4 \mathrm{~Hz}, 2 \mathrm{H}), 0.97(\mathrm{t}, J=7.4 \mathrm{~Hz})$ ${ }^{13} \mathrm{C}\left\{{ }^{1} \mathrm{H}\right\}$ NMR $\left(75 \mathrm{MHz}, \mathrm{CDCl}_{3}\right) \delta: 154.1(\mathrm{dd}, J=289.4,287.7 \mathrm{~Hz}), 133.5(\mathrm{dd}, J=3.4,1.7 \mathrm{~Hz})$, $132.6,131.5(\mathrm{dd}, J=2.9,1.4 \mathrm{~Hz}), 128.12,128.05,127.7,127.5(\mathrm{t}, J=3.4 \mathrm{~Hz}), 126.4,126.4$ $(\mathrm{t}, J=3.2 \mathrm{~Hz}), 126.2,92.5(\mathrm{dd}, J=20.2,14.3 \mathrm{~Hz}), 29.9,21.1(\mathrm{t}, J=2.5 \mathrm{~Hz}), 13.6 .{ }^{19} \mathrm{~F} \mathrm{NMR}$ $\left(282 \mathrm{MHz}, \mathrm{CDCl}_{3}\right) \delta:-92.2(\mathrm{~d}, J=44.1 \mathrm{~Hz}, 1 \mathrm{~F}),-92.4(\mathrm{~d}, J=44.1 \mathrm{~Hz}, 1 \mathrm{~F})$. HRMS (ESI): calcd for $\mathrm{C}_{15} \mathrm{H}_{14} \mathrm{~F}_{2} \mathrm{Ag}(\mathrm{M}+\mathrm{Ag}) 339.0109$, found 339.0105.

(1,1-Difluoro-4,4-dimethylpent-1-en-2-yl)benzene (3j) [25]. Yield $61 \mathrm{mg}(58 \%)$. Colorless oil. Chromatography: hexanes. $\mathrm{R}_{\mathrm{f}} 0.44$ (hexanes). ${ }^{1} \mathrm{H}$ NMR $\left(300 \mathrm{MHz}, \mathrm{CDCl}_{3}\right) \delta: 7.42-7.25$ $(\mathrm{m}, 5 \mathrm{H}), 2.40(\mathrm{dd}, J=3.0,2.1 \mathrm{~Hz}, 2 \mathrm{H}), 0.86(\mathrm{~s}, 9 \mathrm{H}) .{ }^{13} \mathrm{C}\left\{{ }^{1} \mathrm{H}\right\} \mathrm{NMR}\left(75 \mathrm{MHz}, \mathrm{CDCl}_{3}\right) \delta: 154.6$ $(\mathrm{dd}, J=290.5,287.5 \mathrm{~Hz}), 135.8(\mathrm{dd}, J=4.8,2.8 \mathrm{~Hz}), 128.7(\mathrm{t}, J=2.8 \mathrm{~Hz}), 128.4,127.1,91.3(\mathrm{t}$, $J=21.6,13.1 \mathrm{~Hz}), 41.4(\mathrm{~d}, J=1.1 \mathrm{~Hz}), 32.9(\mathrm{dd}, J=3.1,2.2 \mathrm{~Hz}), 29,9 .{ }^{19} \mathrm{~F} \mathrm{NMR}(282 \mathrm{MHz}$, $\left.\mathrm{CDCl}_{3}\right) \delta:-90.7(\mathrm{~d}, J=41.1 \mathrm{~Hz}, 1 \mathrm{~F}),-93.3(\mathrm{~d}, J=41.1 \mathrm{~Hz}, 1 \mathrm{~F})$.

\section{Conclusions}

In summary, we established that potassium xanthogenates can be used for alkylation of $\alpha$-(trifluoromethyl)styrenes leading to gem-difluorinated styrenes under photoredox conditions. Since xanthogenates can be made in one step from alcohols and inexpensive reagents, this reaction provides a convenient method for the generation of alkyl radicals starting from alcohols.

Supplementary Materials: The following are available online at https:/ / www.mdpi.com/article/10 .3390/catal11121555/s1: Copies of NMR spectra.

Author Contributions: V.I.S. experiment; A.D.D. writing. All authors have read and agreed to the published version of the manuscript. 
Funding: This work was supported by the Russian Science Foundation (project 20-13-00112).

Data Availability Statement: The data presented in this study are available in this article.

Conflicts of Interest: The authors declare no conflict of interest.

\section{References}

1. Yan, M.; Lo, J.C.; Edwards, J.T.; Baran, P.S. Radicals: Reactive Intermediates with Translational Potential. J. Am. Chem. Soc. 2016, 138, 12692-12714. [CrossRef]

2. Prier, C.K.; Rankic, D.A.; MacMillan, D.W.C. Visible Light Photoredox Catalysis with Transition Metal Complexes: Applications in Organic Synthesis. Chem. Rev. 2013, 113, 5322-5363. [CrossRef] [PubMed]

3. Neumann, M.; Füldner, S.; König, B.; Zeitler, K. Metal-Free, Cooperative Asymmetric Organophotoredox Catalysis with Visible Light. Angew. Chem. Int. Ed. 2011, 50, 951-954. [CrossRef] [PubMed]

4. Bell, J.D.; Murphy, J.A. Recent advances in visible light-activated radical coupling reactions triggered by (i) ruthenium, (ii) iridium and (iii) organic photoredox agents. Chem. Soc. Rev. 2021, 50, 9540-9685. [CrossRef]

5. Supranovich, V.I.; Levin, V.V.; Kokorekin, V.A.; Dilman, A.D. Generation of Alkyl Radicals from Thiols via Zinc Thiolates: Application for the Synthesis of gem-Difluorostyrenes. Adv. Synth. Catal. 2021, 363, 2888-2892. [CrossRef]

6. Zard, S.Z. On the Trail of Xanthates: Some New Chemistry from an Old Functional Group. Angew. Chem. Int. Ed. 1997, 36, 672-685. [CrossRef]

7. Crich, D.; Quintero, L. Radical chemistry associated with the thiocarbonyl group. Chem. Rev. 1989, 89, 1413-1432. [CrossRef]

8. Tian, F.; Yan, G.; Yu, J. Recent advances in the synthesis and applications of a-(trifluoromethyl)styrenes in organic synthesis. Chem. Commun. 2019, 55, 13486-13505. [CrossRef]

9. Yan, G.; Qiu, K.; Guo, M. Recent advance in the C-F bond functionalization of trifluoromethyl-containing compounds. Org. Chem. Front. 2021, 8, 3915-3942. [CrossRef]

10. Guo, H.-M.; Wu, X. Selective deoxygenative alkylation of alcohols via photocatalytic domino radical fragmentations. Nat. Commun. 2021, 12, 5365. [CrossRef] [PubMed]

11. Speckmeier, E.; Fischer, T.G.; Zeitler, K. A Toolbox Approach to Construct Broadly Applicable Metal-Free Catalysts for Photoredox Chemistry: Deliberate Tuning of Redox Potentials and Importance of Halogens in Donor-Acceptor Cyanoarenes. J. Am. Chem. Soc. 2018, 140, 15353-15365. [CrossRef]

12. Bentrude, W.G. Phosphoranyl radicals-Their structure, formation, and reactions. Acc. Chem. Res. 1982, 15, 117-125. [CrossRef]

13. Bentrude, W.G.; Hansen, E.R.; Khan, W.A.; Min, T.B.; Rogers, P.E. Free-radical chemistry of organophosphorus compounds. III. $\alpha$ vs. $\beta$ Scission in reactions of alkoxy and thiyl radicals with trivalent organophosphorus derivatives. J. Am. Chem. Soc. 1973, 95, 2286-2293. [CrossRef]

14. Rossi-Ashton, J.A.; Clarke, A.K.; Unsworth, W.P.; Taylor, R.J.K. Phosphoranyl Radical Fragmentation Reactions Driven by Photoredox Catalysis. ACS Catal. 2020, 10, 7250-7261. [CrossRef]

15. Hu, X.-Q.; Hou, Y.-X.; Liu, Z.-K.; Gao, Y. Recent advances in phosphoranyl radical-mediated deoxygenative functionalisation. Org. Chem. Front. 2020, 7, 2319-2324. [CrossRef]

16. Maddigan-Wyatt, J.; Hooper, J.F. Phosphorus Compounds as Precursors and Catalysts for Radical C-C Bond-Forming Reactions. Adv. Synth. Cat. 2021, 363, 924-936. [CrossRef]

17. Levin, V.V.; Dilman, A.D. One-pot synthesis of a-trifluoromethylstyrenes from aryl ketones and the Rupper-Prakash reagent. Mendeleev Commun. 2021, 31, 684-685. [CrossRef]

18. He, W.; Ding, Y.; Tu, J.; Que, C.; Yang, Z.; Xu, J. Thermal conversion of primary alcohols to disulfides via xanthate intermediates: An extension to the Chugaev elimination. Org. Biomol. Chem. 2018, 16, 1659-1666. [CrossRef]

19. Akram, R.; Khan, M.D.; Zequine, C.; Zhao, C.; Gupta, R.K.; Akhtar, M.; Akhtar, J.; Malik, M.A.; Revaprasadu, N.; Bhatti, M.H. Cobalt sulfide nanoparticles: Synthesis, water splitting and supercapacitance studies. Mater. Sci. Semicond. Process. 2020, 109, 104925. [CrossRef]

20. Carta, F.; Akdemir, A.; Scozzafava, A.; Masini, E.; Supuran, C.T. Xanthates and Trithiocarbonates Strongly Inhibit Carbonic Anhydrases and Show Antiglaucoma Effects In Vivo. J. Med. Chem. 2013, 56, 4691-4700. [CrossRef]

21. Allwright, E.; Silber, G.; Crain, J.; Matsushita, M.M.; Awaga, K.; Robertson, N. Electrochemical deposition of highly-conducting metal dithiolene films. Dalton Trans. 2016, 45, 9363-9368. [CrossRef]

22. Dumur, F.; Mayer, C.R. Unexpected Ritter Reaction During Acid-Promoted 1,3-Dithiol-2-one Formation. Helv. Chim. Acta 2013, 96, 889-896. [CrossRef]

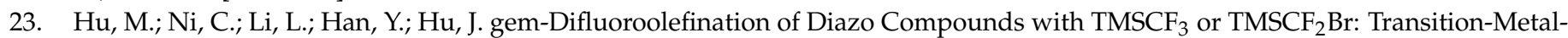
Free Cross-Coupling of Two Carbene Precursors. J. Am. Chem. Soc. 2015, 137, 14496-14501. [CrossRef]

24. Ichikawa, J.; Sakoda, K.; Wada, Y. The 5-endo-trig Cyclization of gem-Difluoroolefins with sp3 Carbon Nucleophiles: Synthesis of 1-Fluorocyclopentenes. Chem. Lett. 2002, 31, 282-283. [CrossRef]

25. Dai, W.; Lin, Y.; Wan, Y.; Cao, S. Cu-Catalyzed tertiary alkylation of $\alpha$-(trifluoromethyl)styrenes with tertiary alkylmagnesium reagents. Org. Chem. Front. 2018, 5, 55-58. [CrossRef] 